\title{
Imaging of individual adatoms on oxide surfaces by dynamic force microscopy
}

\author{
Georg Hermann Simon, ${ }^{1}$ Thomas König, ${ }^{1}$ Hans-Peter Rust, ${ }^{1}$ Maria Verónica Ganduglia-Pirovano, ${ }^{2, *}$ Joachim Sauer, ${ }^{2}$ \\ Markus Heyde, ${ }^{1, \dagger}$ and Hans-Joachim Freund ${ }^{1}$ \\ ${ }^{1}$ Fritz-Haber-Institut der Max-Planck-Gesellschaft, Faradayweg 4-6, D-14195 Berlin, Germany \\ ${ }^{2}$ Institut für Chemie, Humboldt-Universität zu Berlin, Brook-Taylor-Straße 2, D-12489 Berlin, Germany
}

(Received 15 December 2009; published 25 February 2010)

\begin{abstract}
An experimental study of the adsorption sites of metal adatoms on an oxide surface is presented. The adsorption sites of gold on the ultrathin aluminum oxide grown on $\mathrm{NiAl}(110)$ are determined. For the study low-temperature frequency modulation dynamic force microscopy is employed to identify the adsorption sites in real space. Results show that wave trough-like depressions in the alumina surface are preferred locations for gold adspecies.
\end{abstract}

DOI: 10.1103/PhysRevB.81.073411

PACS number(s): 68.37.Ps, 68.47.Gh, 68.43.Bc, 68.43.Fg

Metal adsorption on supported ultrathin oxide films has been studied to advance understanding of metal-oxide interaction. This topic is crucial for a number of technologically important fields. ${ }^{1-5}$ Film thickness is a decisive parameter in these studies because adsorbate properties can depend on it. For example, for gold on $\mathrm{MgO}(100)$ films, the adsorption site depends on the thickness. ${ }^{6}$ In spite of the success of a density-functional theory (DFT) model to describe the aluminum oxide film structure on $\operatorname{NiAl}(110)^{7}$ and its equal success in predicting the bonding mechanism between gold adatoms and the alumina film, the exact adsorption sites of $\mathrm{Au}$ adatoms have not been determined. ${ }^{8}$ One reason for this is the incommensurability of the oxide film along the $\mathrm{NiAl}[001]$ direction, which could not have been fully taken into account by the model calculations. The unit cell of the model alumina structure in the study of $\mathrm{Au}$ adsorption consists of two neighboring oxide unit cells which have been artificially made commensurate to render the periodic calculations practicable. The results show that $\mathrm{Au}$ atoms preferentially bind to surface aluminum $\left(\mathrm{Al}_{\mathrm{S}}\right)$ atoms which are located above an $\mathrm{Al}$ atom of the NiAl substrate. In this case, the $\mathrm{Au}-\mathrm{Al}_{\mathrm{S}}$ bond formation is accompanied by the break of a $\mathrm{Al}_{\mathrm{S}}-\mathrm{O}$ bond within the film and the gain of one electron by the $\mathrm{Au}$. Due to the incommensurability this is possible for all $\mathrm{Al}_{\mathrm{S}}$ sites in the unit cell if a sufficiently large ensemble of unit cells across the surface is probed. In the following, we will show experimentally, that the adsorption sites are always located within the troughs of the oxide film, which are established by the films corrugation and possibly control the diffusibility of the $\mathrm{Au}$ atoms across the surface.

For the present study we employed frequency modulation dynamic force microscopy (FM-DFM) to determine the distribution of actual adsorption sites within the unit cell. Our recent work with the technique on this surface has revealed a contrast of extraordinarily high surface sensitivity [see Fig. 1(a)]. It accurately reproduces the film topography and allows clear identification of the surface oxygen sublattice at high lateral resolution. ${ }^{9,10}$ With this, adsorption sites of single $\mathrm{Au}$ adatoms can be determined with substrate site precision and in principle this can be done for any imageable adsorbed species. Considering the capabilities of FM-DFM on bulk insulators ${ }^{11,12}$ this will enable adatom studies on oxides with spatial resolution equivalent or even surpassing that of scanning tunneling microscopy (STM).
First steps with FM-DFM enabling the imaging of strongly bonded adspecies have been shown. ${ }^{13,14}$ All work presented here has been performed in ultrahigh vacuum at cryogenic temperature $(5 \mathrm{~K})$. The microscope in use is a custom built dual mode STM/FM-DFM machine situated in a helium bath cryostat. Its quartz tuning fork sensor setup records tunneling current and force interaction via the same $\mathrm{Pt} / \mathrm{Ir}$ tip with particular attention paid to the clear separation of the two signals. ${ }^{15}$ Sensor parameters are the unperturbed resonance frequency $\mathrm{f}_{0}=21 \mathrm{kHz}$, a spring constant of $\mathrm{k}$ $\approx 22,000 \mathrm{Nm}^{-1}$, and a quality factor of $\mathrm{Q} \approx 25,000$. In the FM-DFM mode the microscope is operated in the selfexcitation mode at a small constant oscillation amplitude $\mathrm{A}_{\mathrm{OSC}}$ of $380 \mathrm{pm}$. Topography images presented here have been acquired at constant frequency shift $(\Delta \mathrm{f})$. The images had to be straightened out to correct for slight thermal drift. For this the unit cell of the model study in Ref. 7 has been taken as a reference. Preparation of the surface oxide has been done in the usual way. ${ }^{16,17}$

Figure 1(a) shows the atomic surface structure on a terrace (domain $B$ of the two reflection domains) of the ultrathin alumina film that can be grown on $\mathrm{NiAl}(110)$. Unit cell, as well as surface oxygen (large red circles) and surface aluminum (small blue circles) sites from the DFT calculations are indicated. ${ }^{7}$ Green (dark gray) rectangles mark highly recognizable rectangular blocks of eight oxygen sites. Imaging parameters are given in the figure caption. The film consists of four atomic layers and is only $5 \AA$ thick. It is connected to the substrate by interface aluminum sites $\left(\mathrm{Al}_{\mathrm{i}}\right)$ covered with a stack of interface oxygen $\left(\mathrm{O}_{\mathrm{i}}\right)$, surface aluminum $\left(\mathrm{Al}_{\mathrm{S}}\right)$, and surface oxygen $\left(\mathrm{O}_{\mathrm{S}}\right)$ layers. The image in Fig. 1(a) exhibits a characteristic wavelike pattern with the blocks of eight oxygen sites on top of the crests. Corrugations measure up to $60 \mathrm{pm}$ across such a crest and fractions of this parallel to it. Figure 1(b) shows a larger section of such a surface upon deposition of $\mathrm{Au}$ while the surface was at cryogenic temperature. Coverage is about $0.3 \%$ using the $\mathrm{Al}_{\mathrm{S}}$ sites for quantification. The atoms thermalize quickly on the surface and cannot visit larger numbers of sites before eventually coming to rest. Thus, they do not form larger agglomerates but mostly single adsorbed species, sometimes dimers or short chains. This is in agreement with earlier studies. ${ }^{8,18}$ Experimental identification of the actual adsorption sites with STM therein has, however, been difficult. As stated be- 

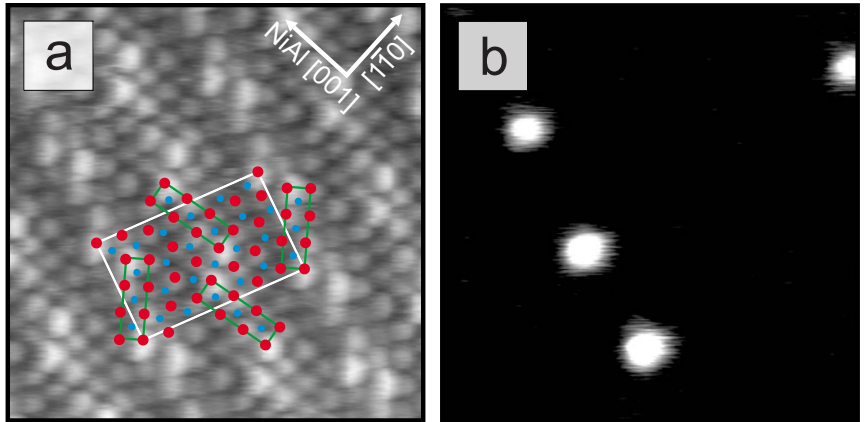

FIG. 1. (Color online) (a) Atomically resolved FM-DFM image of the bare alumina on $\mathrm{NiAl}(110)$ surface. Onto the unfiltered data the unit cell (white quadrangle) and sites of the surface $\mathrm{O}$ (large red/darker gray circles) and surface $\mathrm{Al}$ (small blue/gray circles) layer from a DFT model have been superimposed. (Ref. 7) Green (dark gray) rectangles highlight important structural elements. Imaging parameters: $\Delta \mathrm{f}=-2.75 \mathrm{~Hz}$ and $\mathrm{V}_{\mathrm{S}}=-220 \mathrm{mV}$; scan area: $4.2 \mathrm{~nm} \times 4.2 \mathrm{~nm}$. (b) Image showing adsorbed Au on the alumina film at more moderate imaging conditions: $\Delta \mathrm{f}=-1.9 \mathrm{~Hz}$ and $\mathrm{V}_{\mathrm{S}}$ $=-200 \mathrm{mV}$; scan area: $10 \mathrm{~nm} \times 10 \mathrm{~nm}$.

fore, a FM-DFM contrast with strong surface sensitivity exists, which enables straight forward interpretation. This is particularly true for Fig. 1(a) which could be shown to represent the topography of the terminating oxygen layer of the alumina film on $\mathrm{NiAl}(110)$ with high accuracy. ${ }^{9,10}$ For the determination of individual adsorption sites the position and chemical nature of surface species needs to be clearly identifiable from the obtained images. Here $\mathrm{O}_{\mathrm{S}}$ and $\mathrm{Al}_{\mathrm{S}}$ sites at the surface cannot only be distinguished by their number $\left(\mathrm{O}_{\mathrm{S}}: 28, \mathrm{Al}_{\mathrm{S}}: 24\right)$, but also by their lateral and vertical arrangement. ${ }^{9}$ Hence the imaged protrusions are clearly identified as surface oxygen sites. The $\Delta \mathrm{f}$ set point used to acquire Fig. 1(a) has been chosen to image the substrate with atomic resolution, while the setpoint in Fig. 1(b) has been selected to image the adspecies without lateral manipulation. This is necessary as the interaction during atomic resolution imaging of the oxide is stronger than the barrier for removal of the Au. Scanning across a gold atom with the tip at oxide imaging conditions would simply remove the atom. This is illustrated by the comparison of $\Delta \mathrm{f}(\mathrm{z})$ curves above a gold atom and above the bare oxide (see Fig. 2). In fact the dip in the curve above the $\mathrm{Au}$ adatom does give evidence that already some relaxation event occurred in the tip-sample gap. Consequently the sections which would be suitable for good resolution in $z$ direction, indicated by ellipses for the case of the oxide and the $\mathrm{Au}$, show different interaction strength and are clearly separate. Atomic resolution on the oxide while imaging the $\mathrm{Au}$ is not possible. The imaging conditions have to be set to lower $\Delta \mathrm{f}$ (milder interaction) above the adsorbates if their sites are to be determined. While the atomic resolution scan of the oxide neighborhood approaches an adsorbed $\mathrm{Au}$ atom the tip is then lifted before the Au atom is reached and brought back to the previous imaging conditions behind it. One such measurement is presented in Fig. 3(a). Resolution on the oxide has not been forced to reach a level similar to that in Fig. 1 in order to not put the tip at risk. The superimposed model of the surface oxygen layer in Fig. 3(b)

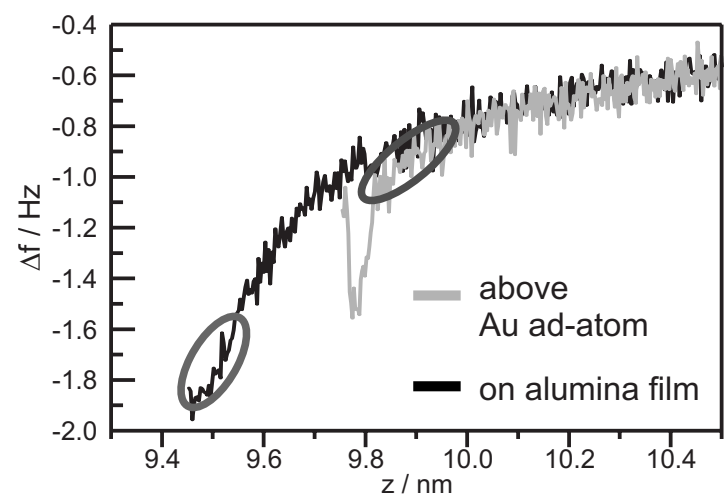

FIG. 2. Frequency shift-distance $[\Delta \mathrm{f}(\mathrm{z})]$ curves above the bare oxide surface and atop a gold atom taken at $\mathrm{V}_{\mathrm{S}}=-300 \mathrm{mV}$. The minimum value of $\Delta \mathrm{f}$ and therefore the interaction differs significantly. Curve sections that are desirable and available for imaging set points lie within the drawn ellipses.

allows now direct identification of the lattice site underneath the adsorbed $\mathrm{Au}$ atom. Prerequisites for this technique are atomic resolution before and after reaching the atom, a stable tip throughout the whole image, low drift and a reproducible $\Delta f(z)$ curve. All of these conditions are fulfilled in atomic resolution FM-DFM measurements so that this type of measurement appears rather easy to implement. Yet one more issue needs consideration, namely, the clear identification of the surface sublattice of each species. This should interestingly be easier to achieve on more complex oxide surfaces with lower symmetry than on highly symmetric facets such as, e.g., (001) terminated rock salt structures. Larger atomically resolved areas, neighboring the adspecies, allow a more precise placement of the structural model and thus better correction of the drift distorted image. In the case of the
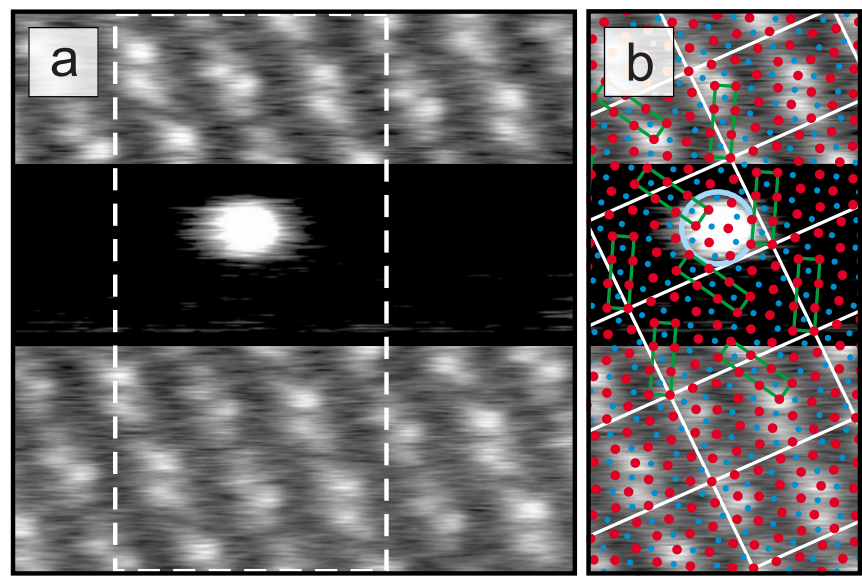

FIG. 3. (Color online) (a) FM-DFM image across a gold atom adsorbed to alumina on $\mathrm{NiAl}(110)$ illustrating the adsorption site determination. The tip has been retracted to a moderate set point at the sharp change in contrast to image the adsorbate and put back to the initial values behind it. (b) Picture detail within the white box in (a) with a superimposed surface lattice (Ref. 7) of the oxide underneath [assignment as in Fig. 1(a)]. The empty light blue (light gray) circle indicates the location of the $\mathrm{Au}$ atom. Imaging parameters: $\mathrm{V}_{\mathrm{S}}=-200 \mathrm{mV}$ and $\Delta \mathrm{f}=-1.5 \mathrm{~Hz}(-0.9 \mathrm{~Hz}$ above the $\mathrm{Au}$ atom $)$. Scan area $6.4 \mathrm{~nm} \times 6.4 \mathrm{~nm}$. 


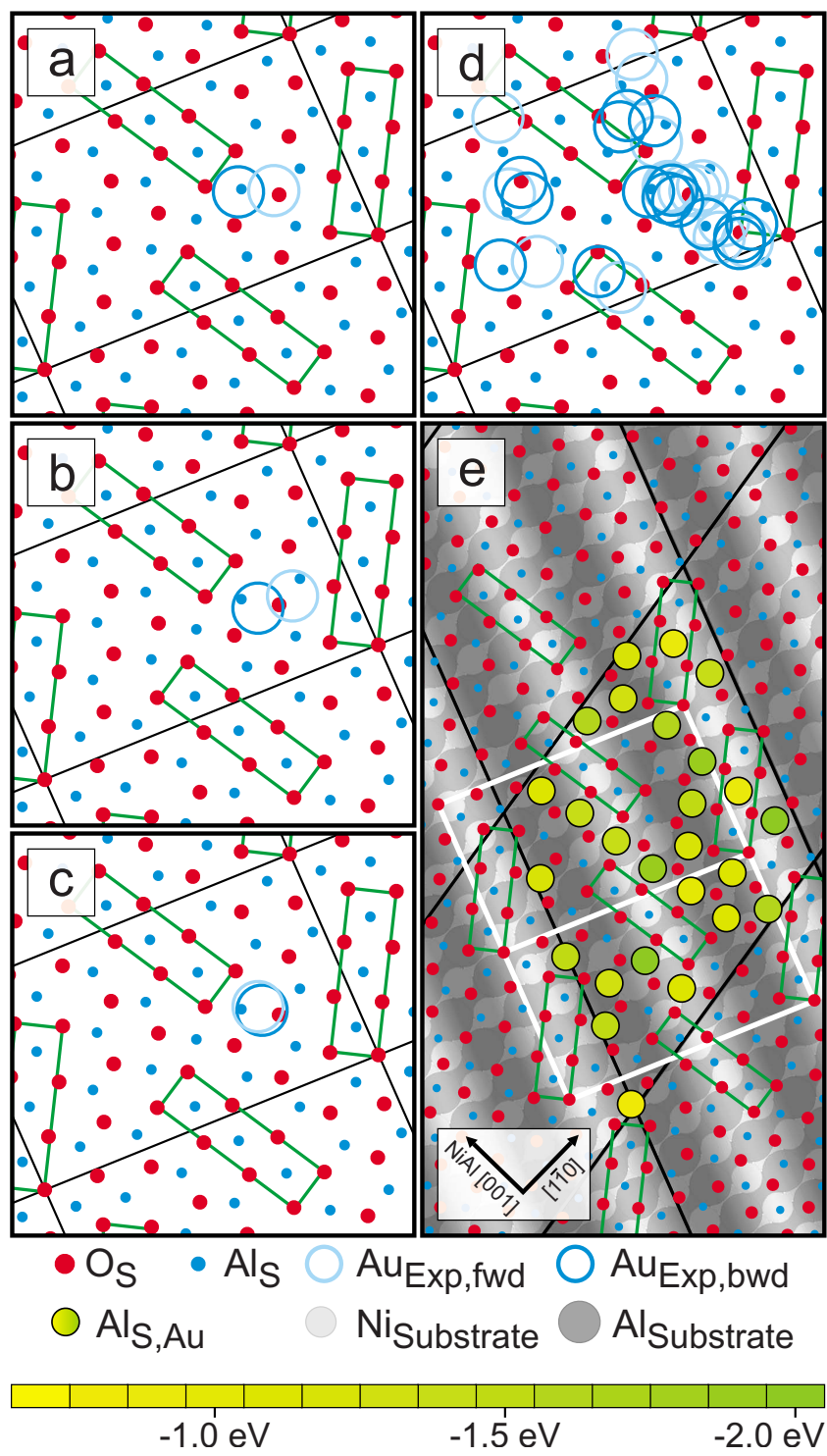

FIG. 4. (Color online) Sites for adsorbed Au species determined by experiment and theory. (a)-(c) FM-DFM results from a series of three images, where the atom appeared in two distinct sites for forward and backward scanning direction at first and eventually stayed on one. Imaging parameters for (a), (b), and (c): $\mathrm{V}_{\mathrm{S}}$ $=-200 \mathrm{mV}, \Delta \mathrm{f}=-1.50,-1.50$, and $-1.58 \mathrm{~Hz}(-0.90,-0.93$, and $-0.93 \mathrm{~Hz}$ above the Au atom). (d) All experimental data forward and backward scans $\left(\mathrm{Au}_{\mathrm{Exp}, \mathrm{fwd}} / \mathrm{Au}_{\mathrm{Exp}, \mathrm{bwd}}\right)$ from the two alumina reflection domains drawn onto the corresponding oxide surface positions in one unit cell. Sizes: (a)-(d) $2 \mathrm{~nm} \times 2 \mathrm{~nm}$. (e) Binding sites [large circles ranging from yellow to green (light gray to gray)] for negatively charged $\mathrm{Au}$ atoms $\left(\mathrm{Al}_{\mathrm{S}, \mathrm{Au}}\right)$ as found by DFT calculations for a parallelogram shaped commensurate supercell (black lines) that covers two oxide unit cells (white quadrangles). For computational details see Ref. 8. The stronger the binding the greener (darker) the circle depicting the adsorption site. A scale is given in the figure legend together with the complete nomenclature. The binding sites are drawn together with the two topmost oxide layers $\left(\mathrm{Al}_{\mathrm{S}}, \mathrm{O}_{\mathrm{S}}\right)$ and the nickel and aluminum atoms of the substrate. The latter are given in the registry used in the calculations and a grayscale wave pattern has been put underneath the two oxide layers to indicate the wave crests and troughs in the oxide's topography. alumina on $\mathrm{NiAl}(110)$ typical double spots that exhibit the same orientation on rows along the direction of the short oxide lattice vector and alternating orientation on neighboring rows along the long lattice vector are a helpful landmark. They indicate two diagonally opposing corners of the rectangular blocks of eight oxygen sites and provide easy placement of the lattice. If the $\mathrm{Au}$ atom is imaged as a circle the position of the adatom can be derived directly with an accuracy that allows identification of the surface lattice site. The shown site lies above a quadrangle of surface oxygen atoms and close to an $\mathrm{Al}_{\mathrm{S}}$ site. The site is in close proximity to one of the rectangular blocks which places it in the deeper stripes lying between the crests formed by those structural elements. We have observed that the site of the adatom can change under the influence of the scanning tip. Figures 4(a)-4(c) show a series where the adsorbate moved between two different sites depending on the fast scan direction (a) and (b), i.e., forward or backward, while it stayed in one position in frame (c). Such behavior may be regarded as a clue that the latter sites is preferential. After all $\mathrm{Au}$ adatoms are predicted to adsorb with a single vertical $\mathrm{Au}-\mathrm{Al}_{\mathrm{S}}$ bond. However the experimental situation could provide another point of view: the occupied site might be one of the many local minima in the complex unit cell, but correspond to a metastable situation. In fact, it has been observed that some atoms are easier displaced than others. The aspects described above demonstrate how the slight freedom of movement affects measurements and how to conclusively identify locations in which $\mathrm{Au}$ adatoms have high probability of being found, namely, within the troughs of the oxide film.

Theoretical treatment of metal atom adsorption on oxide surfaces has found the surface oxygen sites to be favorable in some cases. ${ }^{19,20}$ Yet, the DFT study of Au adsorption onto the particular alumina/NiAl(110) system reports $\mathrm{Al}_{\mathrm{S}}$ sites (above $\mathrm{Al}$ rows of the NiAl) to be favorable. The calculations suggest a mechanism of bond rearrangement in the oxide film upon $\mathrm{Au}-\mathrm{Al}_{\mathrm{S}}$ formation which involves a broken $\mathrm{Al}_{\mathrm{S}}-\mathrm{O}_{\mathrm{i}}$ bond and is accompanied by the transfer of one electron to the $\mathrm{Au}$ atom. Particularly stable binding configurations are found when undercoordinated $\mathrm{O}_{\mathrm{i}}$ is able to form a new bond to an $\mathrm{Al}$ atom in the $\mathrm{NiAl}(110)$ surface. In experiments this registry is unfortunately unknown. Despite the row matching along the commensurate $\mathrm{NiAl}[1 \overline{1} 0]$ direction the actual nature and position of the substrate atom underneath a specific surface site stays unclear and is therefore not drawn in Figs. 4(a)-4(d). Figure 4(d) shows all FM-DFM determined adatom sites from both reflection domains in one unit cell. Most sites appear beside the protruding blocks of oxygen sites. Experimentally $\mathrm{Au}$ atoms are preferentially found in the shallow depressions between the rows of rectangular oxygen blocks. The high lying sites within these blocks are less likely to be occupied and no adsorbate has been found on the quadrangles connecting them on one crest. This finding is in principle reproduced by the DFT calculations for Au adsorption [Fig. 4(e)]. Here a particular registry between film and substrate is given. One finds binding sites for $\mathrm{Au}$ adatoms $\left(\mathrm{Al}_{\mathrm{S}, \mathrm{Au}}\right)$ atop $\mathrm{Al}_{\mathrm{S}}$ sites throughout the oxide unit cell. The blocks of eight oxygen sites, however, are sparsely covered. In fact, most sites and especially the ones with strongest 
binding do lie in the shallow depressions between them. One also sees that slight shifts in registry alter the binding significantly if the two marked oxide unit cells are compared to each other. Among those $\mathrm{Al}_{\mathrm{S}}$ sites that have been found to be special adsorption sites in Ref. 8, the fivefold coordinated sites on the wave crests are less favorable than the fourfold coordinated ones in the wave troughs. For adsorption on fivefold coordinated special $\mathrm{Al}_{\mathrm{S}, \mathrm{Au}}$ binding energies of 1.4$1.6 \mathrm{eV}$ have been calculated compared to $1.5-2.0 \mathrm{eV}$ for fourfold coordinated special $\mathrm{Al}_{\mathrm{S}, \mathrm{Au}}$ sites in the shallow depressions. Adsorption sites without participation of the $\mathrm{NiAl}$ substrate, on the other hand, exhibit Au binding energies below $1.4 \mathrm{eV}$. Another factor influencing the observed binding sites is the diffusion of the Au atoms across the alumina film after deposition. It is conceivable that the corrugation of the alumina film across the unit cell influences the area for preferentially observed binding sites.

In the present study adsorption sites of highly dispersed $\mathrm{Au}$ adatoms on the ultrathin alumina on $\mathrm{NiAl}(110)$ have been determined. The FM-DFM method allows imaging of the $\mathrm{Au}$ adatoms despite the fact that they are easily moved by the scanning tip during atomic resolution imaging of the underlying oxide. It has been shown that images can be recorded that allow the atomic sites of the alumina film underneath the adsorbed Au to be inferred. Together with an unambiguously interpretable contrast the adsorption sites could be determined with atomic resolution. Results show the variety of adsorption sites to be richer than that captured by previous theoretical work. Due to the incommensurability of the alumina film and the NiAl substrate, the found sites should not be interpreted as specific adsorption sites within the oxide unit cell, but rather as locations in which adatoms have high probability of being found. These positions have in common that they preferentially lie within the wave troughs of the alumina film.

It can be anticipated that the FM-DFM method is applicable to other adspecies and to both conducting surfaces and those of bulk insulators. The method has the potential to eventually unlock insulating surfaces for atomic scale real space studies.
*Present address: Institute of Catalysis and Petrochemistry (ICPCSIC), Campus UAM, Cantoblanco, 28049 Madrid, Spain.

†heyde@fhi-berlin.mpg.de

${ }^{1}$ G. Ertl, H. Knözinger, F. Schüth, and J. Weitkamp, Handbook of Heterogeneous Catalysis (Wiley-VCH Verlag $\mathrm{GmbH} \& \mathrm{Co}$. KGaA, Weinheim, 2008), Vol. 4.

${ }^{2}$ H.-J. Freund, Surf. Sci. 500, 271 (2002).

${ }^{3} \mathrm{H}$. Baltes and W. Göpel, Sensors Update (VCH Verlagsgesellschaft mbH, Weinheim, 1996), Vol. 1.

${ }^{4}$ Toyota Institute of Technology, Proceedings of the First Toyota Workshop on Magnetism and Magnetic Materials for High Density Information Storage, edited by A. J. Freeman (Elsevier, Brussels, Belgium, 1997).

${ }^{5}$ P. Chakraborty, J. Mater. Sci. 33, 2235 (1998).

${ }^{6}$ M. Sterrer, T. Risse, M. Heyde, H.-P. Rust, and H.-J. Freund, Phys. Rev. Lett. 98, 206103 (2007).

${ }^{7}$ G. Kresse, M. Schmid, E. Napetschnig, M. Shishkin, L. Köhler, and P. Varga, Science 308, 1440 (2005).

${ }^{8}$ N. Nilius, M. V. Ganduglia-Pirovano, V. Brázdová, M. Kulawik, J. Sauer, and H.-J. Freund, Phys. Rev. Lett. 100, 096802 (2008).

${ }^{9}$ G. H. Simon, T. König, M. Nilius, H.-P. Rust, M. Heyde, and
H.-J. Freund, Phys. Rev. B 78, 113401 (2008).

${ }^{10}$ G. H. Simon, T. König, H.-P. Rust, M. Heyde, and H.-J. Freund, New J. Phys. 11, 093009 (2009).

${ }^{11}$ M. Reichling and C. Barth, Phys. Rev. Lett. 83, 768 (1999).

${ }^{12}$ C. Barth and M. Reichling, Nature (London) 414, 54 (2001).

${ }^{13}$ Y. Sugimoto, M. Abe, S. Hirayama, N. Oyabu, Ó. Custance, and S. Morita, Nature Mater. 4, 156 (2005).

${ }^{14} \mathrm{~S}$. Gritschneder, Y. Iwasawa, and M. Reichling, Nanotechnology 18, 044025 (2007).

${ }^{15}$ M. Heyde, G. H. Simon, H.-P. Rust, and H.-J. Freund, Appl. Phys. Lett. 89, 263107 (2006).

${ }^{16}$ R. M. Jaeger, H. Kuhlenbeck, H.-J. Freund, M. Wuttig, W. Hoffmann, R. Franchy, and H. Ibach, Surf. Sci. 259, 235 (1991).

${ }^{17}$ M. Kulawik, N. Nilius, H.-P. Rust, and H.-J. Freund, Phys. Rev. Lett. 91, 256101 (2003).

${ }^{18}$ M. Kulawik, N. Nilius, and H.-J. Freund, Phys. Rev. Lett. 96, 036103 (2006).

${ }^{19}$ D. Fuks, Y. F. Zhukovskii, E. A. Kotomin, and D. E. Ellis, Surf. Sci. 600, L99 (2006).

${ }^{20}$ S. A. Fuente, P. G. Belelli, R. M. Ferullo, and N. J. Castellani, Surf. Sci. 602, 1669 (2008). 